\section{Fertilität reduzierende Wirkung langdauernder Noradrenalinbehandlung}

Bekanntlich geht der neurale Stress regelmässig mit einem starken Exzess der Nebennierenmarkhormone (Adrenalin und Noradrenalin) parallel. Im Tierreich besteht überdies ein Parallelismus, einerseits zwischen hoher Fertilität und Adrenalingehalt, andererseits zwischen niedrigerer Fertilität und Noradrenalingehalt der Nebennieren ${ }^{1}$. Wie Spencer schon vor 100 Jahren beobachtete ${ }^{2}$, reproduzieren "ängstliche" Tiere in viel höherem Masse als "nicht ängstliche». Dies könnte mit der bekannten Tatsache im Einklang stehen, dass die Angst erregende, neurale Belastung mit Adrenalinämie und die aggressive Reaktion befördernde Belastung mit Noradrenalinämie parallel gehen ${ }^{3,4}$. Der neurale Stress fördert im Ticrexperiment nicht nur die ACTH- und TrH-Produktion der Hypophyse, sondern auch die Cronadotrophin-Aktivität ${ }^{3-7}$. Es schien uns daher angebracht, die Wirkung der Nebennierenmarkhormone auf die Fruchtbarkeit zu untersuchen.

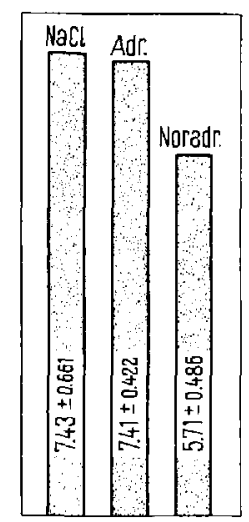

Methodik. Männliche und weibliche weisse Ratten wurden 3 Monate lang mit. Adrenalin oder Noradrenalin behandelt. Die Ticre bekamen das Nebennierenmarkhormon täglich subkutan in steigenden Dosen von 20 bis 150 $\mu \mathrm{g} / \mathrm{kg}$. Die Flüssigkeitsmenge wurde konstant auf $0,2 \mathrm{ml}$ gehalten. Die Kontrolltiere bekamen in gleicher Menge physiologische $\mathrm{NaCl}$. Nach dreimonatiger Behandlung wurde eine 10 tägige Ruheperiode eingeschaltet und nach dieser je ein Rattenmännchen und ein -weibchen nach gleicher Behandlung als Paar abgesondert. Zur Wurfzeit wurde das Weibchen isoliert. Nach Ende der Laktationsperiode wurden die Eltern wieder zusammengebracht, und die Paarung wurde bis zum Ende der Reproduktionsfähigkeit mehrmals wiederholt. 1)ie Zahl der Abkömmlinge wurde registriert. Bei den filialen Generationen wurde das ganze Verfahren wiederholt, das heisst, die Abkömmlinge bekamen dieselbe Behandlung wie die Eltern; so konnte die Fertilität mehrere Generationen hindurch verfolgt werden. Insgesamt wurden 3 Generationen behandelt und die Resultate zusammengefasst ausgewertet. Die beobachtete Wurfaahl erreichte bei der Adrenalingruppe 27, bei der Noradrenalingruppe 31 und bei der NaCl-Gruppe 24.

Resultate. Wie auch an der ligur ablesbar, hat die Adrenalinbehandlung keinen Effekt auf die Fruchtbarkeit der Ratten. Bei der NaCl-Gruppe ist die Zahl der Abkömmlinge pro Wurf $7,43 \pm 0,661$, bei der Adrenalingruppe $7,41+0,422$; das heisst, die Fertilität ist praktisch gleich. Die Noradrenalinbehandlung dagegen hat eine stark reduzierende Wirkung auf die Fruchtbarkeit. Die Zahl der Abkömmlinge pro Wurf beträgt in dieser Gruppe nur 5,71 $\pm 0,486$, was eine signifikante Differenz gegenüber beiden anderen Gruppen bedeutet $(T=2,85$; $\boldsymbol{P}<0,01)$. Trotz der relativ niedrigen Wurfzahl - insgesamt 82 Beobachtungen - konnten wir somit feststellen, dass die 3 Monate dauernde Noradrenalinbohandlung eine reduzierende Wirkung auf dic Fruchtbarkeit der Ratten ausgeübt hat.

Summary. Albino rats of both sexes were treated with epinephrine and norepinephrine for 3 months, while the control animals received the same quantity of physiological $\mathrm{NaCl}$. At the end of this period, the rats were allowed to multiply freely in each group. The number of litters was recorded in each case. While epinephrine did not alter the litter-rate, norepinephrine decreased it significantly. The effect of neural stress on reproduction and its relation to the adrenomedullar hormones is discussed.

\section{A. Sar-HaLÁsz}

Zentral-Institul für Nerven- und Geisteskrankheiten, Budapest-Lipotmezö (Ungarn), 7. September 1964.

1 U. S. von Euler, Noradrenaline (C. Thomas, Springfield 1956). 2 H. Spencer, Die Prinzipien der Biologic, Bd. II (Deutsch) (Schweizcrbart, Stuttgart 1877).

3 D. Funkenstein, S. H. ling und M. Drolette, l'sychosom. Med. 16, 404 (1954).

- I. Elmadjlan, J. M. Hope und F. T. Lamson, J. clin. Endocrinol. $17,608(1957)$.

5 K. Kovacs, A. Jakobovits, M. Divit, Ii. Horvatu, D. BarLaCH und B. Korpassy, Kisérl. Orvostud. 8, 126 (1956).

- S. ARvay und L. Baldzsy, lindokrinol. 37, 276 (1959).

1 A. Saj-Halasz, Proc. lII. World Congre'ss on Fertility and Sterility (Excerpta Med., Ansterdam 1961).

\section{Corticoid and Salicylate Induction of Gastric Acid Secretion in the Vagotomized Rat}

Many observations have been reported which suggest a positive correlation between gastric acid secretion and ulceration. In the case of experimental induction of gastric ulceration by drug administration, this relationship exists for many but not all drugs. Administration of salicylates by various routes has equivocally been described as stimu- latory ${ }^{1-3}$, inhibitory ${ }^{4,5}$, or non-effectual ${ }^{8-8}$ toward gastric acid secretion in mammals. Steroicl derivatives have similarly been described as variable ${ }^{12}{ }^{-12}$, inhibitory ${ }^{13,14}$, or stimulatory 16,18 toward eliciting gastric acid secretion, although the latter effect is most often reported. In addition, the ulcerogenic influence of salicylates 17,18 and corticoids ${ }^{10-21}$ has been established, and these compounds have been shown in our laboratory to be synergistic in this toxic side effect ${ }^{22}$. Thus, the present investigation 